\title{
Carácter emprendedor en estudiantes universitarios: variables demográficas y estrés académico.
}

\section{Entrepreneurial character in university students: demographic variables and}

\section{academic stress.}

\author{
Mario Lado, Sara Rouco \\ Universidade de Santiago de Compostela
}

\begin{abstract}
Resumen
El objeto de este estudio era conocer la influencia de variables demográficas sobre el carácter emprendedor y conocer su relación con el estrés académico. Existe abundante literatura acerca de las características que conforman el perfil del emprendedor y que facilitan su disposición a involucrarse en proyectos complejos; sin embargo, escasean estudios que relacionen el estrés académico con el carácter emprendedor. 336 estudiantes universitarios de diversos cursos y Grados participaron en este estudio. Los resultados indicaron que existen diferencias en el carácter emprendedor en función de la edad y del curso; además, los resultados indicaron que carácter emprendedor y estrés académico son dos variables inversas aunque con correlaciones bajas o moderadas.

Palabras clave: Emprendedor, estrés académico, estudiantes universitarios.
\end{abstract}

\begin{abstract}
The purpose of this study was to know the influence of demographic variables on the entrepreneurial personality and to know the relation with the academic stress. There is a big amount of literature about the characteristics that shape the entrepreneur's profile and that facilitate their willingness to get involved in complex projects; However, there are few studies that relate academic stress to entrepreneurship. 336 university students from various courses and Degrees participated in this study. The results indicated that there are differences in the entrepreneurial character according to age and course; In addition, the results indicated that entrepreneurship and academic stress are two inverse variables, although with low or moderate correlations.
\end{abstract}

Keywords: entrepreneurship, academic stress, university students.

\section{Introducción}

El objeto de este estudio era conocer la influencia de diferentes variables socio demográficas y del estrés académico sobre el carácter emprendedor en una muestra comparativa de estudiantes universitarios.

En las últimas décadas se ha venido prestando cada vez más atención al emprendimiento como una de las vías más adecuadas para hacer frente a la situación de crisis económica, y sus consecuencias laborales y en el desempleo.

Existen diversas definiciones acerca de emprendimiento. El Libro Verde de la Comisión de las Comunidades Europeas lo define como "un modelo mental junto con un proceso de crear y desarrollar una actividad económica combinando con cierto talante gerencial, riesgo de creatividad y/o innovación". Frecuentemente el punto de partida para analizar el emprendedurismo, o el carácter emprendedor, se sitúa en torno a la consideración del carácter emprendedor como un conjunto de características del individuo, atributos y competencias que le facilitan un comportamiento orientado a la acción de emprender, de iniciar una actividad económicamente viable y caracterizada por la autonomía, creatividad, innovación, incertidumbre y riesgo. En ese sentido, las características de personalidad han sido frecuentemente analizadas con el fin de reconocer los rasgos que diferencian el comportamiento de las personas emprendedoras frente a las que no lo son, o no son consideradas como tales; sobre ese asunto, existen recientes meta-análisis que proporcionan evidencia positiva de los rasgos de personalidad (Sánchez, 2009).

Por otra parte, existe otro grupo de estudios que han propuesto otras variables de tipo psicosocial y que intervienen en el perfil del emprendedor: la familia, el espacio sociolaboral y el entorno personal. Un entorno familiar en el que haya personas con vinculación emprendedora facilita el desarrollo de un proyecto emprendedor (Katz, 1992); el espacio sociolaboral también puede facilitar este tipo de desarrollo si ese espacio ha aportado al individuo una experiencia determinada, le ha incorporado conocimientos, competencias para superar dificultades en la realización de las tareas, y si en entorno le presta apoyo social (Krueger, Reilly, \& Carsrud, 2000); y, por último, el propio espacio personal del individuo, y que hace referencia a los rasgos de personalidad, la necesidad de logro, la capacidad de asumir riesgos, la creatividad, la iniciativa y la perseverancia (Moriano, Palací y Morales, 2006). 
La perspectiva de género también ha sido objeto de estudio en emprendedores sin concusiones unánimes; por una parte, algunos estudios concluyen que los varones poseen más cualidades y tienen más probabilidades de tener éxito en los proyectos emprendedores (por ej., Fuentes); por otra parte, otros estudios concluyen que no existen grandes diferencias entre varones y mujeres $\mathrm{y}$, cuando estas existen, se decantan a favor de las mujeres (p.ej., Quevedo, Izar y Romo, 2010).

El estrés, y más concretamente el estrés académico, han sido ampliamente estudiados y existen numerosas publicaciones acerca de su naturaleza y de la influencia en el rendimiento de los estudiantes universitarios (García-Ros, Pérez-González, Pérez-Blasco y Natividad, 2010; Martín, 2007). Entre otras definiciones, el estrés es considerado como un estado de tensión psicológica que se produce cuando los requisitos de la situación exceden a los recursos de la propia persona y le supone a ésta una amenaza para su bienestar personal (Lazarus \& Folkman, 1986). En el ámbito académico, se ha estudiado, por ejemplo, el efecto del estrés sobre el rendimiento académico; en este sentido, ha propuesto que el estrés provoca estados de ansiedad que, a su vez, dificultan la concentración y otros procesos mentales, como la memoria, y disminuye el rendimiento, además de ocasionar trastornos psicosomáticos. Sin embargo, apenas existen estudios que investiguen la influencia del estrés en el carácter emprendedor de los estudiantes universitarios.

El objetivo de este estudio era conocer, por una parte, si las características de los participantes (género, edad, tipo de Grado y curso en que están matriculados) influyen en el carácter emprendedor, y, además, si existe alguna relación entre el carácter emprendedor y el estrés académico.

\section{Método}

\section{Muestra}

Los participantes de este estudio fueron 336 estudiantes universitarios (78 varones y 258 mujeres) que asistían a cursos de Grado en la Universidad de Santiago de Compostela durante el curso 2016-17; el rango de edad oscilaba entre los 18 y los 49 años $(\square=21.00 ; S D=$ 4.05); por facultades, 101 cursaban el Grado en Relaciones Laborales y recursos Humanos, 86 en Magisterio, 80 en Psicología, y 9 un Doble Grado; por cursos, 190 cursaban $1^{\circ}$ y 99 en $4^{\circ}$ de Grado.

\section{Medidas}

Los instrumentos que se utilizaron en este estudio han sido la Escala de Autoeficacia Emprendedora (EAE) y el Cuestionario de Estrés Académico en la Universidad (CEAU). La EAE está compuesta por 23 ítems y se agrupan en cinco factores: Desarrollar nuevos productos, Gestionar los Recursos Humanos, Iniciar relaciones con inversores, Construir un entorno innovador, y Trabajar con estrés. Cada participante contesta al cuestionario indicando en qué grado que considera que sería capaz de desempeñar eficazmente cada una de las tareas indicadas en los ítems, y se responden en una escala de 5 puntos, desde (1) "Totalmente incapaz" hasta (5) "Totalmente capaz”. El CEAU está compuesto por 21 ítems y se agrupan en cuatro factores: Obligaciones Académicas, Expediente y Perspectivas de Futuro, Dificultades Interpersonales y, por último, Expresión y Comunicación de Ideas Propias. Cada participante contesta al cuestionario indicando que nivel de tensión percibe en las situaciones o tareas académicas indicadas en los ítems, y las responden en una escala de 5 puntos, desde (1) "Ninguna tensión" hasta (5) "Mucha tensión".

\section{Procedimiento}

Se solicitó permiso a varios profesores de la Facultad de Relaciones Laborales (Grado en Relaciones Laborales y Recursos Humanos) y de la Facultad de Ciencias de la Educación (Grado de Magisterio) con el fin de administrar los cuestionarios a alumnos de ambas facultades y de diferentes cursos, y en los momentos que ellos consideraran más idóneos para interrumpir lo menos posible sus sesiones. La administración de los cuestionarios se realizó en un plazo de tres semanas, aproximadamente.

\section{Resultados}

El primer análisis consistió en la elaboración de un ANOVA para conocer si el género de los participantes influía en el carácter emprendedor de los mismos. Los resultados indicaron que no existen diferencias significativas entre varones y mujeres en ninguno de los factores. Los resultados se muestran en la Tabla 1.

Tabla 1.

Influencia del Sexo sobre el Carácter Emprendedor.

\begin{tabular}{lrrrrrr}
\hline & \multicolumn{2}{c}{ Varón } & \multicolumn{2}{c}{ Mujer } \\
Factores & $M$ & $S D$ & \multicolumn{1}{c}{$M$} & $S D$ & \multicolumn{1}{l}{$F$} & \multicolumn{1}{l}{$p$} \\
\hline DNP & 13.28 & 2.20 & 13.41 & 2.49 & .16 & .691 \\
GRH & 14.21 & 2.61 & 14.22 & 2.41 & .01 & .951 \\
IRI & 17.72 & 2.41 & 17.53 & 2.78 & .29 & .592 \\
CEI & 13.73 & 2.36 & 13.38 & 2.49 & 1.19 & .276 \\
TBE & 6.51 & 1.29 & 6.49 & 1.34 & .02 & .886
\end{tabular}

Nota $:$ DNP $=$ Desarrollar nuevos productos; GRH $=$ Gestionar recursos humanos; IRI = Iniciar relaciones con inversores; CEI $=$ Construir entornos innovadores; TBE $=$ Trabajar bajo estrés; RL \& RH= Relaciones Laborales y Recursos Humanos.

Se realizó un segundo análisis para comprobar si la edad de los participantes influía en el carácter emprendedor de los mismos. Los resultados indicaron que los participantes con edad superior al promedio puntuaban más alto en todos los factores que los participantes con edad inferior al promedio, aunque estas diferencias solamente eran significativas en dos de los cinco factores, que eran "Desarrollar nuevos productos" y "Construir entornos innovadores". Los resultados se muestran en la Tabla 2. 
Tabla 2.

Influencia de la Edad sobre el Carácter Emprendedor.

\begin{tabular}{lrrrrrl}
\hline & \multicolumn{2}{c}{ Promedio } & \multicolumn{2}{c}{ Promedio } \\
Factores & $M$ & $S D$ & \multicolumn{1}{l}{$M$} & $S D$ & $F$ & $p$ \\
& & & & & & \\
\hline DNP & 13.11 & 2.35 & 13.64 & 2.48 & 4.04 & $.045^{*}$ \\
GRH & 14.08 & 2.42 & 14.36 & 2.48 & 1.05 & .307 \\
IRI & 17.36 & 2.61 & 17.79 & 2.77 & 2.07 & .151 \\
CEI & 13.15 & 2.32 & 13.77 & 2.57 & 5.37 & $.021^{*}$ \\
TBE & 6.37 & 1.28 & 6.62 & 1.35 & 3.02 & .083
\end{tabular}

(*) $p<.05$

Nota $:$ DNP $=$ Desarrollar nuevos productos; GRH $=$ Gestionar recursos humanos; IRI = Iniciar relaciones con inversores; CEI $=$ Construir entornos innovadores; $\mathrm{TBE}=$ Trabajar bajo estrés; RL \& RH= Relaciones Laborales y Recursos Humanos.

Un tercer análisis se realizó para comprobar si el Grado Universitario que cursan los participantes influía en las puntuaciones de los mismos en el carácter emprendedor. Los resultados indicaron que los participantes que cursaban el Grado en Relaciones Laborales y Recursos Humanos obtuvieron puntuaciones más altas en la mayoría de los factores; sin embargo, las diferencias ninguna de las diferencias era significativa. Los resultados se muestran en la Tabla 3.

Tabla 3.

Influencia del Grado sobre el Carácter Emprendedor.

\begin{tabular}{lrrrrrr}
\hline & \multicolumn{3}{c}{ RL \& RH } & \multicolumn{2}{c}{ Magisterio } & \multicolumn{2}{c}{ Psicología } \\
Factores & $M$ & $S D$ & \multicolumn{1}{c}{$M$} & $S D$ & \multicolumn{1}{c}{$M$} & $S D$ \\
& & & & & & \\
\hline DNP & 13.45 & 2.24 & 13.38 & 2.45 & 13.38 & 2.57 \\
GRH & 14.46 & 2.65 & 14.13 & 2.41 & 14.01 & 2.27 \\
IRI & 17.71 & 2.41 & 17.69 & 2.83 & 17.46 & 2.79 \\
CEI & 13.49 & 2.99 & 13.47 & 2.60 & 13.48 & 2.57 \\
TBE & 6.50 & 1.27 & 6.47 & 1.40 & 6.56 & 1.36
\end{tabular}

Tabla 3. (Continuación)

Influencia del Grado sobre el Carácter Emprendedor.

\begin{tabular}{lcccc}
\hline \multicolumn{5}{c}{ Doble Grado } \\
Factores & $M$ & $S D$ & $F$ & $p$ \\
& & & & \\
\hline DNP & 13.00 & 2.00 & .17 & .953 \\
GRH & 14.22 & 1.76 & .49 & .744 \\
IRI & 17.00 & 2.69 & .58 & .680 \\
CEI & 13.22 & 1.79 & .04 & .998 \\
TBE & 6.11 & 1.17 & .27 & .900 \\
\hline
\end{tabular}

Nota $:$ DNP = Desarrollar nuevos productos; GRH = Gestionar recursos humanos; IRI = Iniciar relaciones con inversores; CEI $=$ Construir entornos innovadores; $\mathrm{TBE}=$ Trabajar bajo estrés; RL \& RH= Relaciones Laborales y Recursos Humanos.

Se realizó un cuarto análisis para comprobar si el curso en que los participantes estaban matriculados influía en las puntuaciones en el carácter emprendedor. Los resultados indicaron que los participantes que asistían al $4^{\circ}$ curso obtuvieron puntuaciones más altas en todos los factores que los participantes que asistían a $1^{\circ}$ curso, y esas diferencias eran significativas, a excepción de la diferencia en el factor de "Gestionar los recursos humanos". Los resultados se muestran en la Tabla 4.

Tabla 4.

Influencia del Curso de Grado sobre el Carácter Emprendedor.

\begin{tabular}{lrrrrrl}
\hline & \multicolumn{2}{l}{$1^{\circ}$ Curso } & \multicolumn{2}{c}{$4^{\circ}$ Curso } \\
Factores & \multicolumn{1}{c}{$M$} & $S D$ & \multicolumn{1}{c}{$M$} & $S D$ & $F$ & $p$ \\
& & & & & & \\
\hline DNP & 13.17 & 2.40 & 13.73 & 2.44 & 4.29 & $.039^{*}$ \\
GRH & 14.03 & 2.45 & 14.53 & 2.44 & 3.27 & .071 \\
IRI & 17.31 & 2.65 & 18.01 & 2.72 & 5.29 & $.022^{*}$ \\
CEI & 13.22 & 2.35 & 13.87 & 2.60 & 5.41 & $.021^{*}$ \\
TBE & 6.37 & 1.29 & 6.70 & 1.34 & 4.87 & $.028^{*}$
\end{tabular}

$(*) p<.05$

Posteriormente, se procedió a hallar la correlación de Pearson para comprobar la relación entre ambas variables, el estrés académico y el carácter emprendedor. Se elaboró una matriz de correlaciones que se muestra en la Tabla 5. Los resultados indicaron todas las correlaciones son de signo negativo, con un rango entre $-.01 \mathrm{y}-.18$, y resultaron significativas aquellas correlaciones entre -.12 y -.18 . No existen correlaciones significativas entre el factor "Dificultades interpersonales" perteneciente al Estrés Académico, y los factores de "Desarrollar nuevos productos" y "Gestionar los recursos humanos", éstos pertenecientes al Carácter Emprendedor. Por el contrario, existen correlaciones negativas y moderadas entre los factores de "Obligaciones Académicas", "Experiencias y perspectivas de futuro", y "Expresión y comunicación de ideas", pertenecientes al Estrés académico, con los factores de "Iniciar relaciones con inversores", "Construir entornos innovadores", y "Trabajar bajo estrés", pertenecientes al Carácter Emprendedor. Los resultados se muestran en la Tabla 5.

Tabla 5

Correlaciones entre Carácter Emprendedor y Estrés Académico.

DNP GRH INI CEI TEB OA EPF DIP EC

\begin{tabular}{|c|c|c|c|c|c|c|c|}
\hline DNP & - & & & & & & \\
\hline GRH & -.09 & - & & & & & \\
\hline INI & -.04 & $.55^{* *}$ & - & & & & \\
\hline CEI & -.09 & $.51 * *$ & $.50 * *$ & - & & & \\
\hline TBE & .01 & $.51 * *$ & $.32 * *$ & $.42 * *$ & - & & \\
\hline $\mathrm{OA}$ & .05 & -.07 & $-.12 *$ & $-.15^{* *}$ & $-.18^{*}$ & - & \\
\hline EPF & -.08 & -.01 & $-.13^{*}$ & $-.16^{* *}$ & -.10 & $.51 * *$ & \\
\hline DIP & .09 & .02 & -.01 & -.03 & -.09 & $.39 * * .24 * *$ & \\
\hline ECI & -.01 & -.05 & $-.16 * *$ & $-.14 *$ & $-.14 * *$ & $.30 * * .27 * *$ & $.25^{*}$ \\
\hline
\end{tabular}

(*) $p<.05 ;(* *) p<.01$

Nota $:$ DNP = Desarrollar nuevos productos; GRH = Gestionar recursos humanos; IRI = Iniciar relaciones con inversores; CEI = Construir entornos innovadores; $\mathrm{TBE}=$ Trabajar bajo estrés: $\mathrm{OA}=$ Obligaciones académicas; $\mathrm{EPF}=$ Expediente $\mathrm{y}$ perspectivas de futuro; DIP = Dificultades interpersonales; ECI $=$ Expresión y comunicación de ideas propias. 


\section{Conclusiones}

Los resultados obtenidos en este estudio indican que no existen diferencias por sexos en las puntuaciones obtenidas en la escala de carácter emprendedor; de hecho, las puntuaciones más altas en cada factor se alternan entre varones y mujeres, y ninguna de las diferencias son significativas. De similar manera ocurre con la influencia del tipo de Grado Universitario que estudian los participantes, en las que las diferencias obtenidas por los participantes de los distintos grados no son significativas.

Por otra parte, existen diferencias significativas entre las puntuaciones de los participantes en función de la edad y del curso al que asisten. Los participantes con edad superior a la media puntúan más alto en todos los factores de la escala de carácter emprendedor que los participantes con edad inferior al promedio, aunque las diferencias solamente son significativas en los factores de "Desarrollar nuevos productos" y "construir entornos innovadores". De igual manera, los participantes que cursan $4^{\circ}$ de Grado obtuvieron mayor puntuación en todos los factores de la escala, y todas las diferencias fueron significativas, a excepción del factor "gestionar los recursos humanos". Cabe pensar que existe una relación lógica entre la edad y el curso en que están matriculados los participantes, a pesar de la varianza de edades en los grupos universitarios, superior a otros niveles educativos, y debido, por ejemplo, a la no limitación de edad para matricularse en un curso de algún grado universitario. Con estos resultados se puede deducir que la edad, la experiencia, o la madurez, pueden constituir una variable que favorezca o incremente el carácter emprendedor.

El estrés académico ha sido otra de las variables que se han estudiado por su posible relación con el carácter emprendedor. Los resultados obtenidos concluyeron que estrés académico y carácter emprendedor son dos variables cuyos factores presentan correlaciones entre bajas y moderadas; además esta correlación es inversa, lo que permite concluir, a pesar de que las correlaciones eran entre bajas y moderadas, que estrés académico y carácter emprendedor son dos conceptos antagónicos, de manera que a medida que aumentan las puntuaciones en una variable, descienden en la otra.

\section{Bibliografía}

Fuentes, F. J. y Sánchez-Cañizares, S. M. (2010). Análisis del perfil emprendedor: una perspectiva de género. Estudios de Economía Aplicada, 28(3), 1-28. http://www.redalyc.org/articulo.oa?id=30120334014

García-Ros, R., Pérez-González, F., Pérez-Blasco, J. y Natividad, L. A. (2012). Evaluación del estrés académico en estudiantes de nueva incorporación a la universidad. Revista Latinoamericana de Psicología, $44 \quad$ (2), 143-154. http://www.redalyc.org/articulo.oa?id=80524058011

Katz, J. (1992). A psychological cognitive model of employment status choice. Entrepreneurship Theory and Practice, 17(1), 29-37.

Krueger, N. F., Jr., Reilly, M. D., \& Carsrud, A. L. (2000). Competing models of entrepreneurial intentions. Journal of Business Venturing, 15, 411-432. http://dx.doi.org/10.1016/S0883-9026(98)00033-0

Lazarus, R. S. \& Folkman, S. (1986). Estrés y procesos cognitivos. Barcelona: Martínez-Roca.

Martín, I. M. (2007). Estrés académico en estudiantes universitarios. Apuntes de Psicología, 25(1), 87-99.

Moriano, J. A., Palací, F. J. y Morales, J. F. (2006). El perfil psicosocial del emprendedor universitario. Revista de Psicología del Trabajo y de las Organizaciones, 22(1), 75-99.

Quevedo, L. M., Izar, J. M. y Romo, L. (2010). Factores endógenos y exógenos de mujeres y hombres emprendedores de España, Estados Unidos y México. Investigación y Ciencia, 18(46), 57-63. http://www.redalyc.org/articulo.oa

Sánchez-García, J. C. (2009). Evaluación de la personalidad emprendedora: validez factorial del cuestionario de orientación emprendedora (COE). Revista Latinoamericana de Psicología, 42(1), 41-52. http://www.redalyc.org/articulo.oa?id=80515880004 